\title{
DEFINING LAGRANGIAN IMMERSIONS BY PHASE FUNCTIONS
}

BY

\section{J. ALEXANDER LEES}

\begin{abstract}
In order to analyze the singularities of the solutions of certain partial differential equations, Hörmander, in his paper on Fourier integral operators, extends the method of stationary phase by introducing the class of nondegenerate phase functions. Each phase function, in turn, defines a lagrangian submanifold of the cotangent bundle of the manifold which is the domain of the corresponding differential operator. Given a lagrangian submanifold of a cotangent bundle, when is it globally defined by a nondegenerate phase function? A necessary and sufficient condition is here found to be the vanishing of two topological obstructions; one in the cohomology and the other in the $k$-theory of the given lagrangian submanifold.
\end{abstract}

The object of this paper is to establish necessary and sufficient conditions for the existence and uniqueness of a phase function which globally defines a given immersed lagrangian submanifold $\Lambda$ of the cotangent space $T^{*} M$ of a smooth manifold $M$. For example, the condition for existence is the vanishing of two obstructions, one in $H^{1}(\Lambda ; \mathbf{R})$ and one in $K O^{1}(\Lambda)$. The condition for uniqueness generalizes those given locally by Hörmander and Weinstein. We also establish a covering homotopy property for lagrangian immersions defined by phase functions.

Recall that $T^{*} M$ admits a natural sympletic structure: $\Omega=-d \omega_{M}$, where $\omega_{M}$ is the canonical 1-form on $T^{*} M$. A smooth immersion $\lambda: \Lambda \rightarrow T^{*} M$ is called lagrangian if $\lambda^{*} \Omega=0$, that is, if $\lambda^{*} \omega_{M}$ is closed.

Recall also Hörmander's definition of a nondegenerate phase function: A smooth function $\phi: M \times R^{N} \rightarrow R$ is called a nondegenerate phase function if the smooth map $(x, \alpha) \rightarrow d_{\alpha} \phi(x, \alpha)$ of $M \times R^{N}$ to the fiber $R^{N}$ is nonsingular on the stationary set $\Sigma_{\phi}=\left\{(x, \alpha) \mid d_{\alpha} \phi(x, \alpha)=0\right\}$. Somewhat more generally, we will say that $\phi$ is nondegenerate on a collection of components $\Sigma_{\phi}^{\prime}$ of $\Sigma_{\phi}$ if this condition holds on $\Sigma_{\phi}^{\prime} . \Sigma_{\phi}^{\prime}$ is then a smooth submanifold of $M \times R^{N}$ of dimension $n=\operatorname{dim} M$, and $\lambda_{\phi}: \Sigma_{\phi}^{\prime} \rightarrow T^{*} M$ defined by $\lambda_{\phi}(x, \alpha)$ $=\left(x, d_{x} \phi(x, \alpha)\right)$ is a lagrangian immersion. We say that $\lambda: \Lambda \rightarrow T^{*} M$ is defined by $\phi$ if $\lambda \Lambda=\lambda_{\phi} \Sigma_{\phi}^{\prime}$.

It is shown in [W1, 6.2] that $\lambda: \Lambda \rightarrow T^{*} M$ extends to a symplectic

Received by the editors November 8, 1977.

AMS (MOS) subject classifications (1970). Primary 58G15, 58F05; Secondary 55B15. 
immersion $\hat{\lambda}: T^{*} \Lambda \rightarrow T^{*} M$, where $\Lambda$ is identified with the zero-section $\mathcal{O}(\Lambda) \subset T^{*} \Lambda$. The differential $d \hat{\lambda}$ is thus a bundle map which commutes with the almost complex structures on $T^{*} \Lambda$ and $T^{*} M . d \hat{\lambda} \mid \mathcal{O}(\Lambda)$ thus defines a $U(N)$-equivalence of the complexifications $T \Lambda \otimes \mathbf{C}$ and $(\pi \lambda)^{*} T M \otimes \mathbf{C}$. In terms of classifying maps, $d \hat{\lambda}$ defines a map of $\Lambda$ into $U / O$, regarded as the fiber of the fibration $B O \stackrel{\otimes \mathrm{C}}{\rightarrow} B U$, which is nullhomotopic iff the unitary trivialization of $\left(T \Lambda \otimes(\pi \lambda)^{*} \nu M\right) \otimes C$ is homotopic to the complexification of a real trivialization of $T \Lambda \oplus(\pi \lambda)^{*} \nu M$, where $\nu M$ is the stable normal bundle of $M$. Since $\Omega(U / O) \simeq B O$ by Bott Periodicity [M, 24.6], the map $\Lambda \rightarrow U / O$ defines an element $k(\lambda)$ in $K O^{1}(\Lambda)$.

We now state the main results of this paper:

Theorem 1. Suppose $\lambda: \Lambda \rightarrow T^{*} M$ is a lagrangian immersion. Then $\lambda$ is defined by a nondegenerate phase function iff $\lambda^{*} \omega_{M}=0$ in $H^{1}(\Lambda ; \mathbf{R})$ and $k(\lambda)=0$ in $K O^{1}(\Lambda)$.

Theorem 1 is a generalization of a result of Weinstein (see 4.3).

We will say that the phase functions $\phi=\phi(x, \alpha)$ and $\tilde{\phi}=\tilde{\phi}(x, \tilde{\alpha})$ are equivalent on $\Sigma_{\phi}^{\prime}$ if there is a diffeomorphism $(x, \alpha) \rightarrow(x, \tilde{\alpha}(x, \alpha))$ defined at least on a neighborhood of $\Sigma_{\phi}^{\prime}$ so that $\phi(x, \alpha)=\tilde{\phi}(x, \tilde{\alpha}(x, \alpha))$ there. Note that if $\phi$ is a nondegenerate phase function, so is $\phi+Q$, where $Q$ is a nondegenerate quadratic form in additional fiber variables, and then $\operatorname{Im} \lambda_{\phi}=$ $\operatorname{Im} \lambda_{\phi+Q}$.

THEOREM 2. Suppose $\phi$ and $\tilde{\phi}$ define the same lagrangian immersion $\lambda$ : $\Lambda \rightarrow T^{*} M$, where $\Lambda$ is connected. Then we can find the quadratic forms $Q$ and $\tilde{Q}$ so that $\phi+Q$ is equivalent to $\tilde{\phi}+\tilde{Q}$ on the component of $\Sigma_{\phi}$ which defines $\lambda$.

The local version of this result is due to Hörmander and Weinstein. We now state the covering homotopy property for lagrangian immersions defined by phase functions.

THEOREM 3. Let $\lambda_{l}: \Lambda \rightarrow M$ be a regular homotopy through lagrangian immersions, and suppose that $\lambda_{1}^{*} \omega_{M}=0$ in $H^{1}(\Lambda ; \mathbf{R})$. Suppose also that $\phi$ is nondegenerate on $\Sigma_{\phi}^{\prime}$, and there defines $\lambda_{0}$. Then we can find a nondegenerate quadratic form $Q$ and a homotopy $\phi_{t}$ of $\phi+Q$ so that $\phi_{t}$ is nondegenerate on $\Sigma_{\phi_{t}}^{\prime}$ and there defines $\lambda_{t} \Lambda$.

Alan Weinstein has pointed out the necessity of stabilizing $\phi$ in Theorem 3 , that is, adding the quadratic form $Q$ : With $\phi(x, \alpha)=\alpha^{3} / 3-\alpha+\alpha x^{2}, \operatorname{Im} \lambda_{\phi}$ is the figure $\infty$, but consideration of the Maslov index shows that the figure 8, while obtained from the figure $\infty$ by a regular homotopy through lagrangian immersions, is not defined by a phase function having a single fiber variable. 
I would like to thank Professor Weinstein for many helpful observations, including the identification of the cohomology obstruction $\lambda^{*} \omega_{M}$ which appears in Theorem 3.

1. The covering homotopy property. In this section we will prove Theorem 3. The first step is the proof of a local covering homotopy property:

Lemma 1.1. Let $\lambda: \Lambda \rightarrow T^{*} M$ be a lagrangian immersion, and let $p \in \Lambda$. Then $\lambda$ is defined near $p$ by a nondegenerate phase function $\phi$. If $\lambda_{l}: \Lambda \rightarrow T^{*} M$ is a small regular homotopy of $\lambda$ through lagrangian immersions, we can choose $\phi_{t}$ defining $\lambda_{t}$ near $p$ so that $\phi_{t}$ depends smoothly on $t$.

Proof. The first part of the lemma appears in [H, 3.1.3], but we sketch the proof here for completeness. Recall that the canonical l-form $\omega_{X}$ on $T^{*} X$ is given in local cotangent coordinates $(x, \xi)$ by $\omega_{X}=\Sigma \xi_{i} d x_{i}$.

Now in appropriate coordinates $\left(x^{\prime}, \xi^{\prime}\right)$ about $\lambda p$ obtained from cotangent coordinates $(x, \xi)(x \in X \subset M)$ by a local symplectic equivalence, $\Lambda$ is the graph of a closed 1-form $\theta: \Lambda=\left\{\left(x^{\prime}, \xi^{\prime}\right) \mid \xi^{\prime}=\theta\left(x^{\prime}\right)\right\}$. Then $h:\left(x^{\prime}, \xi^{\prime}-\theta\left(x^{\prime}\right)\right)$ $\rightarrow(x, \xi)$ is symplectic, so $h^{*} \omega_{X^{\prime}}=\omega_{X}-d \phi$, say, where $\phi=\phi(x, \xi)$. On $\lambda \Lambda$, $\omega_{X^{\prime}}=0$, so $d \phi=\omega_{X}$ and, hence, $(x, \xi) \in \Sigma_{\phi}, \lambda_{\phi}(x, \xi) \in \lambda \Lambda$. It is clear that we can choose $\theta$, hence $\phi$, depending smoothly on a parameter $t$ as in the conclusion of the lemma.

Now let $H=D^{k} \times D^{n-k} \subset \Lambda$ be a handle and write $\delta H$ for a neighborhood (say $\partial D^{k} \times D^{n-k+1}$ ) of $\partial D^{k} \times D^{n-k}$ in $H$. Let $p_{H}$ be the basepoint $(-1,0, \ldots, 0) \in \delta H$.

LEMMA 1.2. Let $\lambda_{i}: \Lambda \rightarrow T^{*} M$ be a small isotopy through lagrangian embeddings. Suppose that $H$ is so small that it lies in a neighborhood as in 1.1 with $p=p_{H}$. Suppose $\phi_{t}^{\delta}=\phi_{t}^{\delta}(x, \xi)$ has already been defined as in 1.1, so that $\phi_{t}^{\delta}$ defines $\lambda \mid \delta H$. Then we can define $\phi_{t}$ as in 1.1 so that $\phi_{t}$ extends $\phi_{t}^{\delta}$, provided $k \neq 1$. If $k=1$, there is an $\mathbf{R}$-valued obstruction to doing so.

Proof. Choose $\phi_{t}$ as in 1.1 so that $\phi_{t}\left(p_{H}\right)=\phi_{t}^{\delta}\left(p_{H}\right)$. If $\delta H$ is connected, this determines $\phi_{t}$ so that it agrees with $\phi_{t}^{\delta}$ on a neighborhood of the part of the domain which corresponds to $\delta H$. But when $k=1, \phi_{t}=\phi_{t}^{\delta}$ only on the component corresponding to $-1 \times D^{n}$, so the difference $\phi_{t}-\phi_{t}^{\delta}$ on the component corresponding to $1 \times D^{n}$ is the $\mathbf{R}$-valued obstruction of the statement.

REMARK 1.3. The obstructions defined in the proof of 1.2 enjoy the usual properties of an Eilenberg-Whitney obstruction theory [S, 29].

Remark 1.4 (Weinstein). The obstruction in $H^{1}(\Lambda ; \mathbf{R})$ defined by $\lambda_{t}$ is the cohomology class of $\lambda_{t}^{*} \omega_{M}$.

Recall that a local version of Theorem 2 has been proved by Weinstein [W3]. Weinstein's result follows from a theorem of Hörmander [H, 3.1.6]: 
THEOREM 1.5. Let $\phi=\phi(x, \alpha)$ and $\tilde{\phi}=\tilde{\phi}(x, \tilde{\alpha})$ be phase functions, with $\lambda_{\phi}\left(x_{0}, \alpha_{0}\right)=\lambda_{\tilde{\phi}}\left(x_{0}, \tilde{\alpha}_{0}\right)$. Then $\phi$ and $\tilde{\phi}$ are equivalent near $\left(x_{0}, \alpha_{0}\right)$ and $\left(x_{0}, \tilde{\alpha}_{0}\right)$ iff they define the same lagrangian immersion near these points, $\alpha \in R^{N}$, $\tilde{\alpha} \in R^{N}$ and $\operatorname{sgn} d_{\alpha}^{2}\left(x_{0}, \alpha_{0}\right)=\operatorname{sgn} d_{\tilde{\alpha}}^{2} \tilde{\phi}\left(x_{0}, \tilde{\alpha}_{0}\right)$.

We come now to the main result of this section, which is the proof of Theorem 3.

Proof of Theorem 3. The idea is to use the homotopy lifting property 1.2 together with 1.5 to establish a local homotopy lifting property. By a straightforward construction, the local lifts piece together to define the covering homotopy $\phi_{t}$.

Cover $\Lambda \times I$ by neighborhoods of the form $U \times(t-\varepsilon, t+\varepsilon)$ for which 1.1 holds. Choose a finite refinement $\left\{V_{\mu}\right\}$ and a partition of $I$ into subintervals $I^{\prime}$ so that 1.1 holds on $\bar{V}_{\mu} \times I^{\prime}$. Thus, $\lambda_{t} \bar{V}_{\mu}$ is defined by $\phi_{t}^{\mu}$, say, for $t \in I^{\prime}$. Suppose inductively that $Q_{s}$ has been defined, and $\phi_{t}$ with $t \leqslant s$, so that $\phi_{0}=\phi+Q_{s}$ where $I=\left[s, s^{\prime}\right]$.

By 1.5 we can find a finite covering $\left\{W_{\nu}\right\}$ of $\Lambda$ and equivalences of the restrictions of $\phi_{s}+Q_{\nu}$ and $\phi_{s}^{\mu}+q_{\nu}$ for appropriate quadratic forms $Q_{\nu}$ and $q_{\nu}$.

Now choose a handle decomposition of $\Lambda$ so small that each handle $H$ lies in some $V_{\mu} \cap W_{\nu}$. Since $\lambda^{*} \omega_{M}$ is exact, we can suppose that the $\phi_{t}^{\mu}$ 's satisfy the conclusion of 1.2 for each $H$, using the Eilenberg-Whitney obstruction theory [S, 34.2].

We will replace the $Q_{\nu}$ 's by a single quadratic form. Fix points in the domains of $\phi_{s}+Q_{\nu}$ and $\phi_{s}^{\mu}+q_{\nu}$ which correspond to $\lambda_{s} p_{H}, H \subset V_{\mu} \cap W_{\nu}$. Add to $\phi_{s}$ a negative definite quadratic form $Q_{-}$so that index $\left(\phi_{s}+Q_{-}\right)>$ index $\left(\phi_{s}^{\mu}+q_{\nu}\right)$ at the points corresponding to $\lambda_{s} p_{H}$, for each $H$ (we here write index $\psi$ for index $d_{\alpha}^{2} \psi$ where $\psi=\psi(x, \alpha)$; similarly for rank and signature). Now add to $\phi_{s}^{\mu}+q_{\nu}$ the appropriate negative definite quadratic form $q_{-}^{H}$ so that index $\left(\phi_{s}+Q_{-}\right)=\operatorname{index}\left(\phi_{s}^{\mu}+q_{\nu}+q_{-}^{H}\right)$ at the corresponding points. Finally, add positive definite quadratic forms $Q_{+}$and $q_{+}^{H}$ to $\phi_{s}+Q_{-}$and $\phi_{s}^{\mu}+q_{\nu}+q_{-}^{H}$ so that they all have the same number of variables.

Since $\phi_{s}+Q_{-}+Q_{+}$and $\phi_{s}^{\mu}+q_{\nu}+q_{-}^{H}+q_{+}^{H}$ define the same lagrangian immersion, their ranks are the same by $[\mathbf{H}, 3.1 .4]$. Hence, their signatures are also the same and the local equivalences of $\phi_{s}+Q_{\nu}$ and $\phi_{s}^{\mu}+q_{\nu}$ extend trivially to local equivalences of these phase functions.

Now suppose inductively that for some $\phi_{t}^{\delta}$ and quadratic form $Q_{\delta H}, \phi_{t}^{\delta}$ already defines $\lambda_{l} \delta H$ for $t \in I^{\prime}$, and $\phi_{s^{\prime}}=\phi_{s}+Q_{\delta H}$. Then we can write

$$
\left(\phi_{t}^{\delta}+Q_{-}+Q_{+}\right)(x, \zeta)=\phi_{t}^{\mu}(x, \xi)+\left(q_{\nu}+q_{-}^{H}+q_{+}^{H}\right)(\alpha)
$$

where $\zeta=\zeta(\xi, \alpha), \xi$ and $\alpha$ are fiber variables. By construction, $\phi_{t}^{\mu}$ defines 
$\lambda_{t} H$. Hence, $\phi_{t}^{\delta}+Q_{-}+Q_{+}$extends to $\phi_{t}$ so as to define $\lambda_{t} H$. To complete this step, set $Q_{H}=Q_{\delta H}+Q_{-}+Q_{+}$.

It is clear that these phase functions piece together to define a phase function $\phi_{t}^{\prime}$ for $t \in I^{\prime}$ with, say, $\phi_{s}^{\prime}=\phi_{s}+Q^{\prime}$. Write $\phi_{t}^{\prime}=\phi_{t}+Q^{\prime}$ for $t \leqslant s$ so that $\phi_{0}^{\prime}=\phi+Q_{s}+Q^{\prime}$. By construction, $\phi_{t}^{\prime}$ is nondegenerate on the component defining $\lambda_{l}$. This completes the inductive step, and the proof of Theorem 3.

2. The uniqueness theorem. In this section, we prove Theorem 2 . The argument is a modification of Hörmander's proof in the local case $[\mathbf{H}, 3.1 .6]$. The result will follow immediately from

THEOREM 2.1. Suppose $\phi$ and $\tilde{\phi}$ define the same lagrangian immersion $\lambda$ : $\Lambda \rightarrow T^{*} M$, where $\Lambda$ is connected. Then $\phi$ is equivalent to $\tilde{\phi}$ on the component of $\Sigma_{\phi}$ defining $\lambda$ iff $\phi$ and $\tilde{\phi}$ have the same number of fiber variables, and $\operatorname{sgn} d_{\alpha}^{2} \phi=\operatorname{sgn} d_{\tilde{\alpha}}^{2} \tilde{\phi}$ on that component.

PRoof. We are given $\phi=\phi(x, \alpha), \tilde{\phi}=\tilde{\phi}(x, \tilde{\alpha}) ; \alpha, \tilde{\alpha} \in R^{N}$ and $\lambda_{\phi} \Sigma_{\phi}^{\prime}=$ $\lambda_{\dot{\phi}} \Sigma_{\dot{\phi}}^{\prime}$. We will first show that $\tilde{\phi}$ is equivalent to $\psi$, where $d(\phi-\psi)=0$ on $\Sigma_{\phi}^{\prime}$.

Regard $d_{\phi}: M \times A \rightarrow T^{*}(M \times A)$ as a section. Write $\pi_{M}: T^{*}(M \times A) \rightarrow$ $T^{*} M$ for the natural projection. Then $\lambda_{\phi}=\pi_{M} d_{\phi} \mid \Sigma_{\phi}$. By a calculation, $\pi_{M} \mid d_{\phi} \Sigma_{\phi}$ is a submersion which drops rank by $N$. It follows that $\pi_{M} \mid d_{\phi} \Sigma_{\phi}$ extends to an immersion of a neighborhood of $d_{\phi} \Sigma_{\phi}$ in $d_{\phi}(M \times A)$ to a neighborhood of $\lambda_{\phi} \Sigma_{\phi} \times 0$ in $\lambda_{\phi} \Sigma_{\phi} \times R^{N}$, which commutes with projections on $T^{*} M$.

Similarly for $\tilde{\phi}$. Thus we can find neighborhoods $U$ and $\tilde{U}$ of $\Sigma_{\phi}^{\prime}$ and $\Sigma_{\tilde{\phi}^{\prime}}$, respectively, and immersions $\mu: U \rightarrow \lambda_{\phi} \Sigma_{\phi}^{\prime} \times R^{N}, \tilde{\mu}: \tilde{U} \rightarrow \lambda_{\bar{\phi}} \Sigma_{\phi}^{\prime} \times R^{N}$ with the same image, which extend $\lambda_{\phi}$ and $\lambda_{\dot{\phi}}$, respectively, and which commute with projections on $M$. It follows as in [HP, 7] that $\mu$, together with the local inverses for $\tilde{\mu}$, defines a diffeomorphism $U \rightarrow \tilde{U}$ which pulls back $\tilde{\mu}$ to $\mu$ and commutes with projections on $M$.

By construction, $\Sigma_{\phi}^{\prime}$ corresponds to $\Sigma_{\phi}^{-1}$ under this diffeomorphism, and the pull-back $\psi$ of $\phi$ satisfies $d(\phi-\psi)=0$ on $\Sigma_{\phi}^{\prime}$.

We now show that for $\psi$ sufficiently close to $\phi, d(\psi-\psi)=0$ on $\Sigma_{\phi}^{\prime}$ implies $\phi$ is equivalent to $\psi$ on $\Sigma_{\phi}^{\prime}$. Using Taylor's formula, we can write

$$
\psi=\phi+\sum b_{j k} \frac{\partial \phi}{\partial \alpha_{j}} \frac{\partial \phi}{\partial \alpha_{k}}
$$

near a given point of $\Sigma_{\phi}$. Let

$$
\alpha^{\prime}=\alpha+\sum w_{j k} \frac{\partial \phi}{\partial \alpha_{k}}
$$


As in [H, 3.1.6], we obtain

$$
w_{j k}+\sum w_{\nu j} w_{\mu k} \phi_{\nu \mu}=b_{j k} .
$$

Note that these equations have the solution $w_{j k}=0$ if $b_{j k}=0$. By the implicit function theorem, we can solve these equations uniquely for small $w_{j k}$ if the $b_{j k}$ 's are sufficiently small. We will then also have $\alpha \rightarrow \alpha^{\prime}$ nonsingular, and $b_{j k}$ vanishes along $\Sigma_{\phi}^{\prime}$ since $\psi$ is nondegenerate.

Now choose a handle decomposition $\{H\}$ of a neighborhood of $\Sigma_{\phi}^{\prime}$ so small that each handle lies in a neighborhood in which the $w_{j k}$ 's can be found as above. Suppose inductively that $\psi_{\delta H}$ has been defined, with $\psi_{\delta H}$ equivalent to $\psi$ and $\psi_{\delta H}=\phi$ on $\delta H$. That is, $w_{j k}=0$ on $\delta H$. Then the transformation $\alpha \rightarrow \alpha^{\prime}$ defined locally on $H$ extends over $M \times A$ by replacing $w_{j k}$ by $\chi_{H} w_{j k}$ where $\chi_{H}=1$ on $H$, and supp $\chi_{H}$ lies in a neighborhood of $H$. The pull-back $\psi_{H}$ of $\psi_{\delta H}$ then satisfies $\psi_{H}=\phi$ on $H$ and agrees with $\psi_{\delta H}$ on $\delta H$. Further, $\phi-\psi_{H}$ is small if $\phi-\psi_{\delta H}$ is.

By induction, it follows that if $\psi$ is sufficiently close to $\phi$, then $d(\phi-\psi)=$ 0 on $\Sigma_{\phi}^{\prime}$ implies $\phi$ is equivalent to $\psi$ there. To complete the proof, we will use the hypothesis on the signatures to show that $d(\phi-\psi)=0$ on $\Sigma_{\phi}^{\prime}$ implies $\phi$ is equivalent to $\psi$ there. This follows directly from 3.1.7 of Hörmander [H]: The condition on the signatures implies that there is a homotopy $\psi_{t}$ of $\phi$ to $\psi$ through phase functions nondegenerate on $\Sigma_{\phi}^{\prime}$ with $d\left(\phi-\psi_{t}\right)=0$ there. The conclusion now follows by partitioning $I$ into subintervals on which $\psi_{t}-\psi_{s}$ is sufficiently small.

3. The existence theorem. In this section, we will prove Theorem 1 . We first reformulate the condition on the tangent bundles. Recall the construction of Weinstein [W1, p. 336]: Let $\lambda: \Lambda \rightarrow T^{*} M$ be a lagrangian immersion. Let $d \lambda T \Lambda \oplus W$ be a lagrangian splitting of $T\left(T^{*} M\right) \mid \lambda M$. Define $\psi_{W}: W \rightarrow$ $(d \lambda T \Lambda)^{*}$ by $\psi_{W}(x) y=\Omega(x, y)$ so that $1 \times \psi_{W}: T\left(T^{*} M\right) \mid \lambda M \rightarrow(d \lambda T \Lambda) \oplus$ $(d \lambda T \Lambda)^{*}$ is a $U(n)$-equivalence. Different choices of $W$ give different equivalences, but since the space of lagrangian complements is contractible [W1, p. 337], these equivalences are all homotopic.

In particular, if $\lambda$ extends to a symplectic immersion $\hat{\lambda}$ of a neighborhood of $\theta(\Lambda) \subset T^{*} \Lambda, d \hat{\lambda}: T\left(T^{*} \Lambda\right)\left|\vartheta(\Lambda) \rightarrow T\left(T^{*} M\right)\right| \lambda \Lambda$ is an inverse of $1 \times \psi_{V}$ with $V=J d \lambda T \Lambda$, where $J$ is the complex structure on $T\left(T^{*} M\right)$. The unitary trivialization of $\left(T \Lambda \oplus(\pi \lambda)^{*} \nu M\right) \otimes \mathbf{C}$ determined by $\hat{\lambda}$ as in the introduction is homotopic to the complexification of a real trivialization of $T \Lambda \oplus(\pi \lambda)^{*} \nu M$ iff

$$
d \hat{\lambda} \times 1: T\left(T^{*}\left(\Lambda \times R^{N}\right)\right)\left|\Theta(\Lambda) \times 0 \rightarrow T\left(T^{*}\left(M \times R^{N}\right)\right)\right| \lambda \Lambda \times 0
$$

is homotopic to the complexification of a real equivalence $T\left(\Lambda \times R^{N}\right) \rightarrow$ $T\left(M \times R^{N}\right)$. This, in turn, holds iff the stabilization $1 \times \psi_{W}$ of $1 \times \psi_{V}$ is 
homotopic to the complexification of a real equivalence, where

$$
W=J d(\lambda \times 1) T\left(M \times R^{N}\right) \text {. }
$$

We now show that the two conditions mentioned in Theorem 1 are necessary:

THEOREM 3.1. Let $\phi: M \times R^{N} \rightarrow R$ be a nondegenerate phase function. Then $\lambda_{\phi}^{*} \omega_{M}=d\left(\phi \mid \Sigma_{\phi}\right)$ and $\phi$ determines an $O(n+N)$-equivalence of $T\left(\Sigma_{\phi} \times R^{N}\right)$ and $\left(\pi \lambda_{\phi} \times 1\right)^{*} T\left(M \times R^{N}\right)$ whose complexification is the stabilization of the $U(n)$-equivalence of $T \Sigma_{\phi} \otimes \mathbf{C}$ and $\left(\pi \lambda_{\phi}\right) * T M \otimes \mathbf{C}$ determined by $\lambda_{\phi}$.

Proof. The fact that $\lambda_{\phi}^{*} \omega_{M}=d\left(\phi \mid \Sigma_{\phi}\right)$ is already mentioned in [H] and [W2]. Here is a quick sketch of the argument: The result is clear if $\lambda_{\phi}=d \phi$ : $M \rightarrow T^{*} M(N=0)$. Hence, it holds for $d \phi: M \times R^{N} \rightarrow T^{*}\left(M \times R^{N}\right)$. Projecting $T^{*}\left(M \times R^{N}\right)$ onto $T^{*} M$ by $\pi_{M}$ as in Theorem 2, and restricting $\pi_{M} d \phi$ to $\Sigma_{\phi}$, we obtain $\lambda_{\phi}^{*} \omega_{M}=d\left(\phi \mid \Sigma_{\phi}\right)$.

For the second part of the theorem, suppose, to begin with, that $\lambda_{\phi}=d \phi$ : $M \rightarrow T^{*} M$. Then $\pi^{*} T M \mid \lambda M \rightarrow d \lambda T M$ induced by the projection $\pi: T\left(T^{*} M\right)$ $\rightarrow T M$ is an $O(n)$-equivalence which complexifies to an equivalence $T\left(T^{*} M\right) \mid \lambda M \rightarrow(d \lambda T M) \oplus(d \lambda T M)^{*}$. Since the space of lagrangian complements to the bundle along the fibers in $T\left(T^{*} M\right)$ is contractible, this last equivalence is homotopic to $1 \times \psi_{W}$, where $W=J d \lambda T M$.

Now with $M$ replaced by $M \times R^{N}$, the equivalence $1 \times \psi_{W}(W=$ $\left.J d(d \phi) T\left(M \times R^{N}\right)\right)$ is homotopic to the complexification of an $O(n+N)$ equivalence. As in Theorem $2, \lambda_{\phi}=\pi_{M} d \phi \mid \Sigma_{\phi}$, and $\pi_{M}$ is covered by the bundle isomorphism $T\left(T^{*}\left(M \times R^{N}\right)\right) \rightarrow T\left(T^{*} M\right) \oplus T\left(T^{*} R^{N}\right)$ induced by translation. Also, the equivalence $1 \times \psi_{W} \mid d \phi \Sigma_{\phi}$ corresponds under this isomorphism to the stabilization of $1 \times \psi_{V}$, where $V=J d \lambda_{\phi} T \Sigma_{\phi}$. Hence, this stabilization is homotopic to the complexification of an $O(n+N)$ equivalence, and the second condition of 3.1 holds.

Finally, we show that the conditions of Theorem 1 are sufficient:

THEOREM 3.2. Let $\lambda: \Lambda \rightarrow T^{*} M$ be a lagrangian immersion, with $\lambda^{*} \omega_{M}$ exact. Suppose that the map $\Lambda \rightarrow U / O$ defined in the introduction is nullhomotopic. Then $\lambda$ is defined by a phase function.

Proof. Choose a handle decomposition of $\Lambda$. We will define $\phi$ inductively: We will say that $\phi$ is defined over a union $K \subset \Lambda$ of handles if $\phi$ defines $\lambda K$. It will be convenient to perturb $\phi$ defined over $K$ as necessary so as to place $\Sigma_{\phi}$ in general position. By 3.1 and the covering homotopy property of Theorem 3, this can be done without loss of generality.

Choose for each handle $H=D^{k} \times D^{n-k}$ the basepoint $q_{H}=(0,0)$. By $1.1, \lambda$ is defined by a phase function near $q_{H}$. By Theorem $3, \lambda H$ is defined by a phase function $\phi_{H}$. By the remarks in the preceding paragraph, we may 
suppose $\lambda H$ is transverse to the fibers near $\lambda q_{H}$, so $\phi_{H}=f_{H}+Q_{H}$ over a neighborhood of $\lambda q_{H}$.

Now let $H$ be a 1-handle, and suppose $\phi_{H}$ and $\phi_{\delta H}$ have already been defined as above. There is an obvious obstruction to extending $\phi_{\delta H}$ to a phase function equivalent to $\phi_{H}$, namely, the difference of the quantity $\left(f_{H}-f_{\delta H}\right)$ over the two components of $\delta H$. As in the proof of Theorem 3, these differences define an obstruction cocycle, which represents the class of $\lambda^{*} \omega_{M}$ in $H^{1}(\Lambda, \mathbf{R})$. Since this class is assumed to vanish, we can redefine the $f_{\delta H}$ 's so that these obstructions vanish.

There is also a less obvious obstruction to extending $\phi_{\delta H}$, namely, the difference of the quantity (sgn $Q_{H}-\operatorname{sgn} Q_{\delta H}$ ) over the two components of $\delta H$. That this is an obstruction follows from the fact that for any two phase functions $\psi$ and $\psi^{\prime}$ defining the same lagrangian immersion, sgn $\psi-\operatorname{sgn} \psi^{\prime}$ is locally constant ( $\operatorname{sgn} \psi$ as in the proof of Theorem 3). If ( $\operatorname{sgn} Q_{H}-\operatorname{sgn} Q_{\delta H}$ ) is the same over both components of $\delta H$, we can add quadratic forms of signature zero as necessary so that $\phi_{\delta H}$ is equivalent to the restriction of $\phi_{H}$.

One can check that the differences (sgn $Q_{H}-\operatorname{sgn} Q_{\delta H}$ ) define a cocycle satisfying the properties of an Eilenberg-Whitney obstruction theory. The obstruction in $H^{1}(\Lambda ; \mathbf{Z})$ represented by this cocycle is in fact the characteristic class defined by Arnol'd [A], by the calculation in [H, 3.2].

Now suppose inductively that $\phi$ has already been defined over $K$ and let $H$ be a $(j+1)$-handle with $\delta H \subset K$. Then $\phi_{H}$ and $\phi$ are equivalent (after stabilizing) over $\delta H$. Thus, there is a fiber preserving diffeomorphism of a neighborhood of $\lambda_{\phi_{H}}^{-1} \delta H$ with a neighborhood of $\lambda_{\phi}^{-1} \delta H$ which transforms the restriction of $\phi_{H}$ to that of $\phi$. The problem is to extend this to a fiber preserving diffeomorphism of the domain of $\phi_{H}$ (once this has been done, we can use general position to ensure that the new $\Sigma_{\phi}$ is embedded).

By the covering homotopy property of Theorem 3, we may suppose, by shrinking $H$ to a neighborhood of $q_{H}$, that $\lambda_{\phi_{H}} H$ is transverse to the fibers of $T^{*} M$. Thus the problem is to extend over $H$ a map of $\delta H$ into the group $\mathscr{D}_{0}\left(R^{N}\right)$ of diffeomorphisms of $R^{N}$ which preserve the origin. With the compact-open topology, $\mathscr{D}_{0}\left(R^{N}\right)$ has $O(N)$ as a deformation retract [S']. So the obstruction to extension is determined by a map $\delta H \rightarrow O(N)$.

We can regard the product of this map and the trivial map on the first factor of $R^{n} \times R^{N}$ (corresponding to the differential of the projection of $H$ on $M)$ as the restriction to $\delta H$ of the $O(n+N)$-equivalence of $T(\Lambda \times$ $\left.R^{N}\right) \mid K$ and $(\pi \lambda \mid K)^{*} T\left(M \times R^{N}\right)$ determined by $\phi$ as in the beginning of this section. That is, $\delta H \rightarrow O(n+N) \rightarrow U(n+N)$ is nullhomotopic, and a nullhomotopy of $\delta H \rightarrow O(n+N)$ is a lift of this trivialization. With these identifications, $\delta H \rightarrow O(N)$ determines an element of $\pi_{j}(U / O)$.

One can check that the $\pi_{j}(U / O)$ valued cochain defined in this way 
satisfies the properties of an Eilenberg-Whitney obstruction theory. Hence, the vanishing of a sequence of obstructions in $H^{j+1}\left(\Lambda ; \pi_{j}(U / O)\right)$ implies that $\lambda$ is defined by a phase function. Also, the obstructions are the obstructions to finding a nullhomotopy of the map $\Lambda \rightarrow U / O$ defined in the introduction and at the beginning of this section. Finally, the vanishing of the first obstruction implies the vanishing of the Arnol'd characteristic class, by Remark 4.2 below. This completes the proof of 3.2 and of Theorem 1 .

4. Some applications. We first give another formulation of Theorem 1. Given a lagrangian immersion $\lambda: \Lambda \rightarrow T^{*} M$, the differential $d \lambda$ defines a lagrangian distribution $T \Lambda \rightarrow T\left(T^{*} M\right)$ which covers $\lambda$. This distribution can be regarded as a section of $\lambda^{*} l\left(T^{*} M\right)$, where $l\left(T^{*} M\right)$ is the bundle of lagrangian planes in $T\left(T^{*} M\right)$.

Write $\delta \lambda: T \Lambda \rightarrow T\left(T^{*} M\right)$ for the trivial distribution which sends each tangent plane at $p \in \Lambda$ to the lagrangian plane $J F_{\lambda p}$, where $F_{\lambda p}$ is the plane tangent to the fiber of $\pi: T^{*} M \rightarrow M$ at $\lambda p$. In what follows, we will stabilize distributions $\gamma: T \Lambda \rightarrow T\left(T^{*} M\right)$ to distributions $T\left(\Lambda \times R^{N}\right) \rightarrow T\left(T^{*}(M \times\right.$ $\left.R^{N}\right)$ ), and write $\gamma \times 1$ for the stabilization of $\gamma$.

THEOREM 4.1. Let $\lambda: \Lambda \rightarrow T^{*} M$ be a lagrangian immersion. Then $\lambda$ is defined by a phase function iff $\lambda^{*} \omega_{M}=0$ in $H^{1}(\Lambda ; \mathbf{R})$ and $d \lambda$ is stably homotopic to $\delta \lambda$ through lagrangian distributions.

Proof. We first prove necessity: $\lambda^{*} \omega_{M}$ is exact, by 3.1. If $\lambda=d \phi$, the fact that $d \lambda$ and $\delta \lambda$ are homotopic follows from the fact that the space of lagrangian distributions transverse to the bundle along the fibers in $T^{*} M$ is contractible. If $\lambda$ is defined by a phase function, we may write $\lambda=\lambda_{\phi}=$ $\pi_{M} d \phi \mid \Sigma_{\phi}$. Now observe that $d(d \phi)$ is homotopic to the stabilization of $d \lambda_{\phi}$, hence $d \lambda_{\phi}$ and $\delta \lambda_{\phi}$ are stably homotopic.

For the sufficiency: A homotopy of $d \lambda \times 1$ and $\delta \lambda \times 1$ defines a homotopy of $d \lambda \times 1$ through bundle maps to one which sends each tangent plane at $p \in \Lambda$ to the lagrangian plane $J F_{\lambda p}$. Complexify this bundle map to obtain an equivalence of $T\left(\Lambda \times R^{N}\right) \otimes C$ and $(\pi \lambda \times 1)^{*} T\left(M \times R^{N}\right) \otimes C$ which is homotopic to the stabilization of $d \hat{\lambda}$ as in the introduction. By Theorem $1, \lambda$ is now defined by a phase function.

REMARK 4.2. The first obstruction to finding a stable homotopy of $d \lambda$ and $\delta \lambda$ is the characteristic class defined by Arnol'd [H, 3.3] (also compare the conjecture in [W2, 6-6]).

Corollary 4.3 (WeINSTEIn [W2, 6-3]). Let $\lambda: \Lambda \rightarrow T^{*} M$ be a lagrangian immersion with $\lambda^{*} \omega_{M}$ exact, and suppose there is a lagrangian distribution along $\lambda$ in $T\left(T^{*} M\right)$ which is transverse to both $d \lambda T \Lambda$ and the bundle along the fibers of $T^{*} M$. Then $\lambda$ is defined by a phase function. 
Proof. Since the space of lagrangian distributions transverse to the distribution of the hypothesis is contractible, there is a homotopy of $d \lambda$ through lagrangian distributions to $J \delta \lambda$. Now compose this with the linear homotopy of $J$ and $I$ and apply 4.1.

Corollary 4.4 (Stability). Suppose $H^{1}(\Lambda ; \mathbf{R})=0$. Let $\lambda: \Lambda \rightarrow T^{*} M$ be $a$ lagrangian immersion defined by a phase function. Then any lagrangian immersion sufficiently close to $\lambda$ is also defined by a phase function.

Proof. Observe that the fiber $U(n+N) / O(n+N)$ of $(\lambda \times 1)^{*} l\left(T^{*}(M \times\right.$ $\left.R^{N}\right)$ ) is locally contractible and apply 4.1 .

REMARK 4.5. Corollary 4.4 also follows from Theorem 3 and the results of [L].

\section{REFERENCES}

[A] V. I. Arnol'd, On a characteristic class which enters in quantization conditions, Functional Anal. Appl. 1 (1967), 1-13.

[H] L. Hörmander, Fourier integral operators. I, Acta Math. 127 (1971), 79-183.

[HP] A. Haefliger and V. Poenaru, La classification des immersions combinatoires, Inst. Hautes Études Sci. Publ. Math. 23 (1964), 75-91.

[L] J. Lees, On the classification of lagrange immersions, Duke Math. J. 43 (1976), 217-224.

[M] J. Milnor, Morse theory, Ann. of Math. Studies No. 51, Princeton Univ. Press, Princeton, N. J., 1963.

[S] N. Steenrod, The topology of fibre bundles, Princeton Univ. Press, Princeton, N. J., 1951.

[S'] T. E. Stewart, On groups of diffeomorphisms, Proc. Amer. Math. Soc. 11 (1960), 559-563.

[W1] A. Weinstein, Symplectic manifolds and their lagrangian submanifolds, Advances in Math. 6 (1971), 329-346.

[W2] , Lectures on symplectic manifolds, CBMS Regional Conf. Ser. in Math., No. 29, Amer. Math. Soc., Providence, R. I., 1977, pp. 1-48.

[W3] _ Singularities of families of functions, Differentialgeometrie in Grossen (W. Klingenberg, ed.), Bibliographisches Institut, Mannheim, 1971, pp. 323-330.

Department of Mathematics, Duke University, Durham, North Carolina 27706

Current address: Schlumberger Well Services, 5000 Gulf Freeway, P. O. Box 2175, Houston, Texas 77001 